\title{
Individuation Test for Emerging Adults (ITEA): Adaptação para a população portuguesa
}

\author{
Filipa Nunes ${ }^{1,2}$, Filipa Correia ${ }^{3}$ \& Catarina Pinheiro Mota ${ }^{2,3}$ \\ ${ }^{1}$ Faculdade de Psicologia e Ciências da Educação da Universidade do Porto \\ ${ }^{2}$ Centro de Psicologia da Universidade do Porto \\ ${ }^{3}$ Universidade de Trás-os-Montes e Alto Douro
}

\begin{abstract}
Resumo: 0 presente estudo procura adaptar para a população portuguesa o Individuation Test for Emerging Adults (ITEA) e analisar as suas características psicométricas. A amostra em estudo foi constituída por 432 adultos emergentes portugueses com idades compreendidas entre os 18 e os 30 anos. A análise fatorial confirmatória revelou índices de ajustamento adequados confirmando o modelo conceptual original do ITEA. Os resultados alcançados revelam propriedades psicométricas que confirmam a adequação linguística e cultural do ITEA na população portuguesa. 0 ITEA revelou-se capaz de identificar diferenças no processo de distanciamento psicológico aos pais, por parte dos adultos emergentes, em função da idade e género dos participantes. A confiabilidade foi avaliada através do alpha de Cronbach, que revelou índices de .80 /.82 para a totalidade do instrumento na sua versão da mãe e do pai. Para além disto, foi encontrada a invariância de medição entre os papéis parentais e o género do participante.
\end{abstract}

Palavras-chave: Individuação; adultos emergentes; ITEA.

Individuation Test for Emerging Adults (ITEA): Adaptation for the Portuguese population: The present study aims to adapt to "Individuation Test for Emerging Adults (ITEA)" to the Portuguese population and to analyze its psychometric properties. The sample was constituted by 432 emerging adults aged ranging from 18 to 30 years. The confirmatory factorial analysis showed appropriate adjustment indexes had been confirming the original conceptual model of ITEA. The results reveal psychometrics properties that confirm the cultural and linguistic adequacy of ITEA in the Portuguese population. The ITEA was able to the identifying differences in psychological distancing process the emerging adults to the fathers based on the participants' age and gender. The reliability was evaluated through the Alpha de Cronbach that revealed indices the $.80 / .82$ for the instrument totality in the mother and father version, respectively. Furthermore, measurement invariance was analyzed among parental roles and the participant's gender.

Keywords: Individuation; emerging adults; ITEA.

De acordo com os trabalhos desenvolvidos por Margaret Mahler (1990), durante os três primeiros anos de vida a criança realiza um processo de dissolução da fusão simbiótica estabelecida com as suas figuras de referência. Este processo de separação psicológica possibilita à criança a sua autocompreensão como um ser individual e singular distinto das figuras significativas de afeto.

Partilhando desta conceção, Blos (1967) acrescenta que o processo de individuação é contínuo e dinâmico e que, portanto, não se esgota na infância. 0 autor sugere que as relações estabelecidas na díade adolescentes-pais se vão transformando progressivamente com o próprio processo de separaçãoindividuação não perdendo relevância, mas assumindo um caracter mais igualitário e interdependente. Para este autor, o distanciamento psicológico dos pais evolui para estágios mais avançados do desenvolvimento, nomeadamente, a adolescência em consequência da progressiva capacidade de autorregulação da população juvenil. Desta feita, é atribuída a este período desenvolvimental a conotação de "segunda individuação". Tendo por base uma perspetiva psicanalítica, compreende-se que na adolescência ocorre uma reestruturação das representações internas formuladas na infância acerca dos pais enquanto a principal fonte de apoio, afeto e autorregulação. Por esta altura, os adolescentes iniciam um processo de confiança progressiva face aos seus próprios recursos internos o que, por sua vez, poderá

\footnotetext{
${ }^{1}$ Morada para correspondência: Universidade de Trás-os-Montes e Alto Douro. Departamento de Educação e Psicologia, Edifício da Escola de Ciências Humanas e Sociais - Polo I, 5001-801, Vila Real, Portugal. Email: catppmota@utad.pt. Esta investigação é parcialmente suportada pela FCT de acordo com o projecto PEst-C/PSI/UI0050/2011 e FEDER fundos do programa COMPETE inserido no projecto FCOMP-01-0124FEDER-022714
} 
potenciar um sentido de individualidade (Blos, 1967). O estabelecimento de ligações afetivas com as figuras cuidadoras e com os demais sugerem também, ser pautadas pela comunicação recíproca, disponibilidade emocional e apoio mútuos (Koepke \& Denissen, 2012; Youniss \& Smollar, 1985). Cabe destacar, que este processo de individuação, na adolescência, aporta uma significativa ambivalência entre o desejo interno de alcançar a autonomia e independência e a necessidade de permanecer sob o suporte e apoio da esfera familiar (Josselson, 1980).

Embora o conceito de segunda individuação tenha sido, inicialmente, preconizado para a investigação da separação psicológica no decurso da adolescência (e.g., Kruse \& Walper, 2008; Puklek Levpušček, 2006) atualmente é, também, alargado para estudar a associação entre a individuação e o ajustamento psicoafetivo de adultos emergentes (e.g., Buhl, 2008; Correia \& Mota, 2016; Lamborn \& Groh, 2009).

De acordo com Arnett (2007) a idade adulta emergente consiste no período da adolescência que ocorre a partir dos 18 anos e caracteriza-se pela ambiguidade em alcançar um self estável e independente e explorar a formação identitária nas áreas das relações íntimas e da vida profissional. Partilhando desta conceção, Buhl e Lanz (2007) destacam que as mudanças sociais, económicas e demográficas do último século implicaram um maior investimento no percurso académico e formação profissional, denotando-se uma dependência financeira dos pais mais prolongada e um adiamento da saída da habitação familiar. A este fenómeno pode acrescer a prorrogação do processo de separação-individuação (Tanner, 2006). Note que os adultos emergentes ao se aproximarem de papéis sociais tidos como adultos detêm uma maior responsabilidade, autonomia e autocontrolo que possibilita uma progressiva reestruturação das suas relações afetivas com os pais elevando-as para interações entre adultos iguais. Todavia, a entrada na adultícia emergente não traduz o término do processo de independência pessoal, na medida em que, os jovens continuam em certa medida dependentes do apoio familiar permanecendo, não raras vezes, a habitar na residência dos pais e a depender financeiramente destes (Komidar, Zupančič, Sočan, \& Puklek Levpušček, 2013).

De acordo com Lamborn e Groh (2009), o processo de distanciamento psicológico às figuras parentais no decurso da adultícia emergente assume uma vivência menos ambivalente do que na adolescência, na medida em que por essa altura, o contexto familiar propicia mais autonomia e liberdade pessoal, o que se pode rever numa maior capacidade de autodeterminação. Desta feita, a separação psicológica neste período assume implicações distintas face à adolescência embora mantenha proximidade no que concerne à dependência económica e social.

Apesar do reconhecimento desta diferenciação pela comunidade científica, escassos são os instrumentos de medida construídos e formulados especificamente para avaliar este processo na adultícia emergente. De realçar que para a população portuguesa não se conhece, mesmo, nenhum instrumento validado ou adaptado cujo modelo conceptual original tenha sido preconizado para avaliar o processo de separação-individuação em adultos emergentes. Atualmente têm-se assistido a inúmeros processos de adaptação para os jovens adultos de medidas psicométricas formuladas inicialmente para adolescentes. O Munich Individuation Test of Adolescence (MITA; Walper, Schwarz, \& Jurasic, 1996) tem sido utilizado de forma parcial (apenas algumas dimensões) em amostras de adultos emergentes portugueses apresentando índices satisfatórios de consistência interna, assim como índices ajustados nas análises confirmatórias, tanto na versão do pai como na da mãe (Mota \& Matos, 2013). Apesar disto, salienta-se que tais resultados decorrem da utilização parcial do MITA, o que sugere que grande parte das dimensões que contempla a sua versão original não se ajustem totalmente à vivência do processo de individuação dos adultos emergentes portugueses. A este respeito, Josselson (1980) acrescenta que os questionários conceptualizados originalmente para avaliar a individuação na adolescência englobam aspetos do processo de distanciamento e proximidade imaturos que, não raras vezes, são vivenciados e resolvidos pela população juvenil, e como tal, não se ajustam às vicissitudes da adultícia emergente.

Partindo desta conceção, considera-se relevante a realização de análises metodológicas que possibilitem a adaptação, para a população portuguesa, de instrumentos de medida preconizados para avaliar o processo de separação-individuação em adultos emergentes. Neste âmbito, sugere-se o Individuation Test for Emerging Adults (ITEA) de Komidar et al. (2013) cujo modelo conceptual contempla as vicissitudes inerentes à reestruturação das relações entre adultos iguais na tríade mãe-pai-adulto emergente. Na formulação de itens para o ITEA, os autores tiveram em consideração as mudanças ecológicas do desenvolvimento contemplando vivências de comunicação recíproca e de interesse, assim como experiências de diferenciação dos pais e de autodeterminação. Os resultados obtidos nos estudos de validação deste instrumento, realizados numa amostra eslovena, revelaram bons índices de consistência interna em todas as dimensões: Procura de suporte $(\alpha=.92, .90)$; Intrusividade $(\alpha=.91, .83)$, Autoconfiança $(\alpha=.84, .80)$, Medo de desapontar os pais $(\alpha=.87, .87)$, e Ligação $(\alpha=.90 ; .90)$ na versão da mãe e do pai respetivamente. 0 instrumento apresentou, também, bons índices de ajustamento (CFI = 
$.92 / .91, \mathrm{TLI}=.92 / .91, \mathrm{RMSEA}=.07 / .07, \chi^{2} / \mathrm{df}=3.74, \chi^{2} / \mathrm{df}=3.76$ ) tanto na versão da mãe como na do pai, respetivamente. Estes resultados asseguram a confiabilidade e validade do instrumento sugerindo-se o ITEA como um instrumento válido para a avaliação do processo de individuação aos pais de adultos emergentes eslovenos.

Desta forma, o presente estudo assume como principal objetivo adaptar para a população portuguesa o ITEA, assim como analisar as suas características psicométricas. É, igualmente, objetivo deste estudo analisar a invariância do ITEA entre os papéis parentais e o género dos participantes.

\section{MÉTODO}

\section{Participantes}

No estudo participaram 432 jovens adultos com idades compreendidas entre os 18 e os 30 anos (M = 20.77; $D P=2.96)$, dos quais 358 (82.9\%) são do género feminino e 74 (17.1\%) são do género masculino. Relativamente à escolaridade, 5 (1.2\%) frequentavam o 12. ano de escolaridade 166 (384\%) frequentavam o 1. o ano da licenciatura, 97 (22.5\%) o 2. ano da licenciatura, 99 (22.9\%) o 3.․ano da licenciatura, $16(3.7 \%)$ o 1. - ano do mestrado, e $16(3.7 \%)$ o 2. ano do mestrado. Quanto aos estudantes universitários, 106 (24.5\%) frequentavam Psicologia, 41 (9.5), 41 (9.5) Serviço social, 4 (0.9\%) Engenharia Civil, 74 (17.1) Educação Básica, 39 (9.0\%) Engenharia Agronómica, 10 (2.3\%) Engenharia Florestal, 37 (8.6\%) Reabilitação Psicomotora, 48 (11.1\%) Teatro, e 35 (8.1\%) Ciências da Comunicação (33 valores omissos). No que se refere ao estado civil dos jovens adultos, 406 (94.0\%) são solteiros, 23 (5.3\%) são casados ou vivem em união de facto e $3(0.7 \%)$ são divorciados ou separados. Quanto às relações amorosas dos participantes, 252 (58.3\%) têm atualmente um relacionamento amoroso com uma duração média de 2 anos e 9 meses $(M=2.9 ; D P=2.36), 152(35.2 \%)$ já tiveram uma relação amorosa com uma duração média de 1 ano e 4 meses $(M=1.4 ; D P=.87), 22(5.1 \%)$ nunca tiveram algum tipo de relacionamento e $6(1.4 \%)$ mantêm um relacionamento amoroso, mas sem compromisso.

\section{Instrumentos}

O ITEA de Komidar et al. (2013) foi utilizado com o objetivo de avaliar o processo de individuação dos jovens adultos face às figuras parentais. Trata-se de uma escala de autorrelato composta por 36 itens tanto na versão da "Mãe" como na do "Pai". No que se refere à organização do questionário, este apresenta-se segundo 5 fatores, nomeadamente: Procura de suporte, Ligação, Intrusividade, Autoconfiança e Medo de desapontar os pais.

A dimensão Procura de suporte, constituída por 6 itens $(4,8,10,25,31,35)$, pressupõe a compreensão das figuras parentais, por parte dos jovens adultos, enquanto uma base segura de apoio e suporte face à resolução das dificuldades e vicissitudes quotidianas experienciadas (e.g., Quando eu estou com problemas nas minhas relações pessoais eu peço-lhe conselhos). A Ligação, composta por 6 itens $(1,13$, $16,19,22,27)$, define uma série de experiências desenvolvidas na tríade mãe-pai-indivíduo pautadas pela comunicação clara e aberta, compreensão e confiança (e.g., Ele(a) respeita as minhas vontades). A Intrusividade, constituída por 8 itens $(3,6,15,20,29,32,34,36)$, descreve comportamentos de excesso de controlo e preocupação, assim como de invasão da privacidade e intimidade do indivíduo por parte das figuras parentais (e.g., Eu acho que ele(a) quer saber demasiado sobre mim). A Autoconfiança, composta por 8 itens $(2,7,9,12,18,23,30,33)$, descreve atitudes e condutas dos indivíduos pautadas pela autossuficiência, autonomia, independência e maturidade (e.g., Eu tomo decisões sobre a minha carreira, independentemente da opinião dele(a)). Por último, a dimensão Medo de desapontar os pais, constituída por 8 itens $(5,11,14,17,21,24,26,28)$, compreende a preocupação e o receio dos jovens face à sua eventual incapacidade para corresponder às expectativas que as figuras parentais têm face a si (e.g., Quando eu faço algo de errado, preocupo-me com a reação dele(a)).

Cada item tem 5 possibilidades de resposta, apresentadas numa escala tipo likert que varia entre 1Completamente falso e 5-Completamente verdadeiro. 0 maior resultado obtido em cada dimensão sugere a presença de uma maior perceção dos jovens adultos quanto à frequência das situações descritas. Níveis elevados de ligação, procura de suporte, intrusividade e medo de desapontar os pais são considerados disfuncionais na medida em que poderão traduzir uma certa dependência emocional e funcional face às figuras parentais, assim como uma certa invasão por parte destas últimas. Por outro lado, níveis elevados de autoconfiança são considerados positivos ao sugerir uma vivência emocional segura e autossuficiente (Komidar et al., 2013).

Cabe destacar que o ITEA já foi utilizado em amostras de adultos emergentes portugueses, tendo apresentado boas características psicométricas, nomeadamente bons índices de consistência interna e de ajustamento tanto na versão da mãe como na do pai, respetivamente (Correia \& Mota, 2016). 


\section{Procedimento}

Num primeiro momento, solicitou-se a autorização dos autores da versão original do ITEA, para a sua utilização e consequente tradução e adaptação para a população portuguesa. Posteriormente, realizou-se a tradução do instrumento para a língua portuguesa, tendo este processo respeitado todos os critérios definidos internacionalmente para a tradução de instrumentos (Hambleton, 2005). Cabe realçar, que a tradução dos itens da versão original do ITEA para a língua portuguesa foi realizada por tradutores bilingue (português - inglês), sendo à posteriori realizado um exercício de reconversão para o inglês. Foi realizada uma análise por uma equipa de dois especialistas na área da psicologia do desenvolvimento no sentido de garantir o valor semântico e a equivalência linguística e cultural. Foi, ainda, realizada uma reflexão falada com jovens adultos entre os 18 e os 30 anos de idade, o que permitiu verificar que os itens se encontravam percetíveis em termos formais e semânticos e ainda a duração da aplicação requerida, avaliada em cerca de 10 minutos com jovens em contexto universitário, não tendo sido necessária qualquer alteração.

Este estudo fez parte de um projeto de investigação no âmbito da psicologia clínica aprovado pelo Comitê de Ética da Universidade de Trás-os-Montes e Alto-Douro. A recolha de dados foi realizada, num único momento do tempo, no período de outubro a dezembro de 2013, numa instituição de Ensino Superior da região norte de Portugal. Foi realizada uma reunião com a Direção da referida instituição, a quem foi solicitada a devida autorização e clarificados os objetivos, estrutura e pertinência do estudo. Após a aceitação da proposta, os participantes consentiram a aplicação dos questionários, através da assinatura do Termo de Consentimento Livre e Esclarecido. A recolha de dados decorreu em contexto de sala de aula, na presença do investigador responsável que, de forma sucinta, realizou uma série de instruções standard onde foram explicitados os objetivos gerais do estudo, assim como garantidos todos os pressupostos de voluntariedade, privacidade, anonimato e confidencialidade das informações prestadas.

\section{Estratégias de análise de dados}

A presente investigação assume um cariz transversal dado que o conjunto de medições foi realizado num único momento do tempo não existindo, portanto, um seguimento temporal dos indivíduos. A amostragem é não-probabilística, tendo sido recolhida por conveniência, no contexto de uma instituição a norte do país que se manifestou disponível para a participação na presente investigação. O tratamento dos dados foi realizado com o programa estatístico Statistical Package for Social Sciences (SPSS, 20.0) e com IBM SPSS AMOS 25. Numa primeira fase, e no sentido de identificar e excluir missings, e eventuais outliers realizou-se, de forma preliminar, uma limpeza da amostra. Este procedimento de "limpeza da amostra" consiste na exclusão de questionários incompletos ou considerados inadequados por apresentar mais de $10 \%$ de dados ausentes por instrumento. Com o intuito de identificar elementos cujas respostas se distanciavam da média, realizaram-se análises de outliers multivariados mediante o cálculo da distância de Mahalanobis, optando-se por eliminar os indivíduos que se desviavam significativamente da média geral de respostas. Para verificar se os dados seguiam os pressupostos de normalidade, foram analisados os valores de skeweness (assimetria) e kurtosis (achatamento) procedendo-se, concomitantemente, à realização do teste de Kolmogorov-Smirnov e à interpretação dos gráficos de Histogramas, Q-QPlots, Scatterplots e Boxplots (Field, 2005). Os valores calculados confirmaram que a amostra em estudo cumpria os critérios de normalidade procedendo-se, neste sentido, a análises estatísticas mediante testes paramétricos.

$\mathrm{Na}$ continuidade, analisou-se o ajustamento do modelo conceptual original do instrumento aos dados da amostra através da realização de Análises Fatoriais Confirmatórias de 1, a o ordem (AFC) com recurso ao método de estimação de máxima verosimilhança. A AFC consiste num procedimento estatístico que possibilita a testagem da estrutura fatorial dos instrumentos, garantindo uma elevada fiabilidade e validade dos mesmos (Klein, 2005). Na AFC foram analisados diferentes índices de ajustamento, nomeadamente: Comparative Fit Index (CFI), Tucker-Lewis Index (TLI), e Root Mean Square Error of Approximation (RMSEA). Os valores de CFI e TLI iguais ou superiores a .90 representam um modelo aceitável, enquanto os valores de RMSEA iguais ou inferiores a .08 correspondem a um ajustamento aceitável. A proporção do $\chi 2 / \mathrm{df}$ é considerada adequada quando os valores variam entre $1 \mathrm{a}$ 5 (Kline, 2005). Numa fase posterior, foram calculados os coeficientes de alpha de Cronbach do constructo geral e de cada subdimensão em particular do ITEA, que permitiram analisar os valores referentes à homogeneidade da variância dos itens do instrumento (Cronback, 2004).

Para se analisar a intensidade e a direção da associação entre as variáveis escalares do ITEA, foram calculados os coeficientes de correlações de Pearson ( $r$ ) (Field, 2005). A interpretação dos valores obtidos seguiu os seguintes prossupostos: valores de $r=0$ até $r=.20$ indicam uma correlação de magnitude fraca, de $r=.21$, até $r=.50$ de magnitude moderada e $r>.50$ de magnitude forte. Foi, também, analisada a 
invariância de medida deste instrumento quanto aos papéis parentais e ao género dos participantes através de análises multigrupo. Nas comparações entre modelos, utilizaram-se os critérios propostos por Cheung e Lau (2012), sendo eles $\Delta$ CFI $\leq .01$ e $\triangle$ RMSEA <.015. Estes critérios indicam que quando a variação do CFI e do RMSEA entre um modelo mais restrito e o precedente na sequência de invariância apresentam valores inferiores aos pontos de corte acima mencionados, a hipótese de invariância não deve ser rejeitada (Cheung \& Lau, 2012).

Por último foram efetuadas análises de variância uni e multivariada (ANOVA e MANOVA) com o intuito de verificar a presença de diferenças significativas no processo de individuação à mãe e ao pai em função das dimensões sociodemográficas da amostra, nomeadamente idade e género. Para as comparações múltiplas entre os grupos, foram efetuados testes post-hoc recorrendo-se ao teste de Scheffé.

\section{RESULTADOS}

\section{Validade fatorial}

Com o intuito de testar o ajustamento dos dados da presente amostra ao modelo conceptual original do ITEA, realizaram-se AFC de 1. ${ }^{a}$ ordem através do IBM SPSS AMOS 25. Tendo em consideração o elevado número de itens do ITEA (36 na versão da mãe e 36 itens da versão do pai), o que conduzia a um elevado número de parâmetros a estimar, utilizou-se o método de emparcelamento (parcelling) de forma aleatória (designados Suporte 1, Suporte 2, Suporte 3, Ligação 1, Ligação 2, ..., Medo de desapontar 3), tal como propõem autores como Barrett e Kline (1981). Denotar que o método de emparcelamento aleatório, embora seja um pouco controverso (e.g., Levine, Petrides, Davies, Jackson \& Howell, 2005), detém um vasto e extenso corpo teórico a sustentá-lo (e.g., Coffman \& McCullum, 2005).

Tal como se pode verificar na Tabela 1, o modelo de cinco fatores proposto por Komidar et al. (2013) replica-se na amostra portuguesa em estudo. Os resultados sugerem índices de ajustamento de acordo com os valores teoricamente esperados tanto na versão da mãe como na do pai, respetivamente (Figura 1 e 2).

Tabela 1. Ajustamento do ITEA na amostra portuguesa em estudo $(n=432)$

\begin{tabular}{ccccc}
\hline ITEA & $\chi \mathbf{2} / \mathbf{d f}$ & CFI & TLI & RMSEA (IC 90\%) \\
\hline Mãe & 2.91 & .95 & .92 & .07 \\
Pai & 3.02 & .95 & .93 & .07 \\
\hline
\end{tabular}

Nota. CFI - Comparative Fit índex; TLI - Tucker-Lewis Index. RMSEA - Root Mean Square of Error Aproximation.

\section{Confiabilidade}

A análise de consistência interna, na amostra em estudo, demonstrou valores de alpha de Cronbach de .80 /.82 para a totalidade do instrumento na sua versão para mãe e para o pai, respetivamente. Os valores de alpha, relativamente às dimensões, foram igualmente ajustados em ambas as versões parentais (Tabela 2), o que revelam a boa fidedignidade do instrumento na amostra em estudo.

Tabela 2. Alphas de cronbach do ITEA na amostra em estudo comparativamente com os valores do estudo original

\begin{tabular}{|c|c|c|c|c|}
\hline \multirow[b]{2}{*}{ ITEA } & \multirow[b]{2}{*}{ Número de itens } & \multicolumn{2}{|c|}{ Amostra em estudo } & \multirow{2}{*}{$\begin{array}{c}\text { Versão original } \\
\text { Mãe }\end{array}$} \\
\hline & & Mãe & Pai & \\
\hline Procura de suporte & 6 & .85 & .85 & .92 \\
\hline Ligação & 6 & .85 & .88 & .90 \\
\hline Intrusividade & 8 & .84 & .82 & .91 \\
\hline Autoconfiança & 8 & .82 & .83 & .84 \\
\hline Medo de desapontar os pais & 8 & .77 & .84 & .87 \\
\hline Escala total & 36 & .80 & .82 & .77 \\
\hline
\end{tabular}




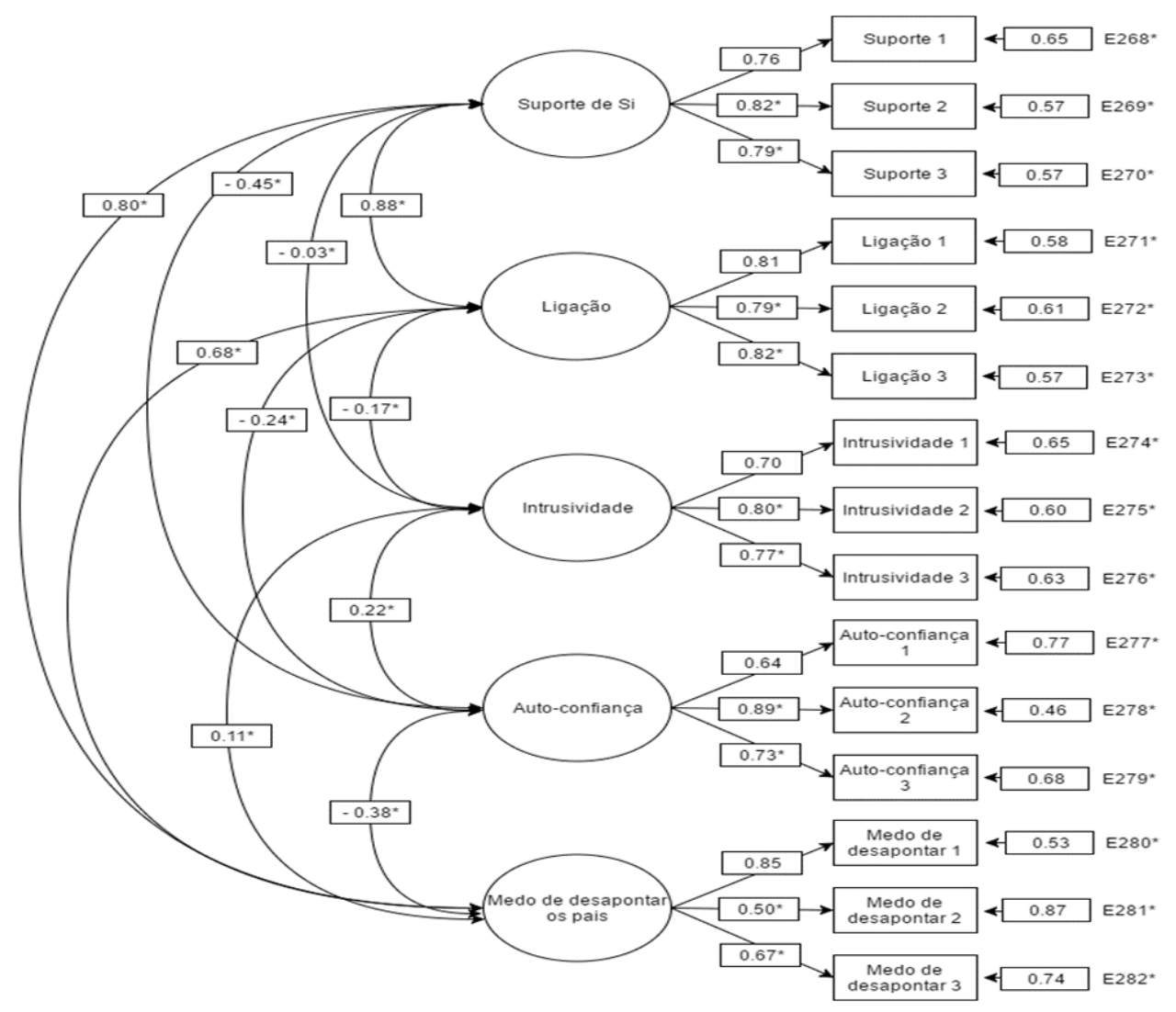

Figura 1. Modelo Fatorial de 1ํo Ordem Testado na Amostra em Estudo (ITEA_Mãe)

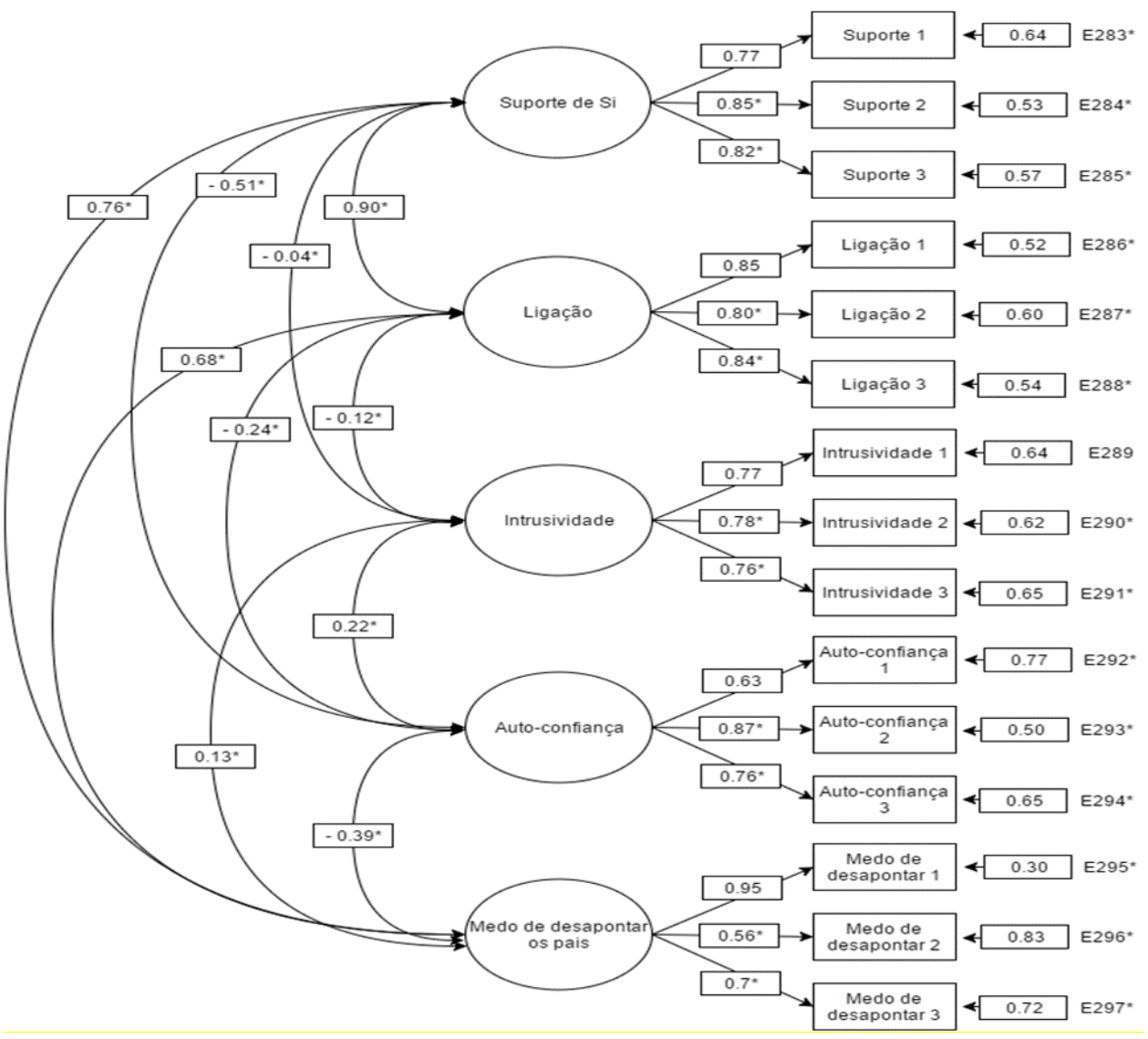

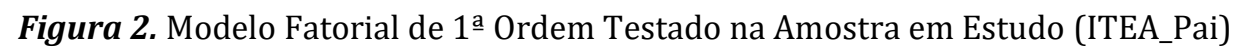




\section{Correlações entre as dimensões do ITEA}

Os resultados das análises de correlações entre as dimensões do ITEA e as respetivas médias e desvios-padrão, reportados na Tabela 3, permitem constatar que existem correlações significativas entre as dimensões que constituem o ITEA. No que concerne à associação entre as dimensões que constituem o ITEA em relação à mãe, os resultados indicam que a dimensão procura de suporte regista uma correlação significativa no sentido positivo e com magnitude forte com a ligação $(r=.73, p=.001)$ e com o medo de desapontar à mãe $(r=.54, p=.001)$ e magnitude moderada com a autoconfiança $(r=-.36, p=.001)$. Por sua vez, a ligação correlaciona-se significativamente no sentido negativo com magnitude fraca com a intrusividade $(r=-.15, p=.001)$ e a autoconfiança $(r=-.18, p=.001)$ e no sentido positivo com magnitude moderada com o medo de desapontar a mãe $(r=.46, p=.001)$. Relativamente à intrusividade, esta revela uma associação significativa no sentido positivo com magnitude fraca com a autoconfiança $(r$ $=.20, p=.001)$ e o medo de desapontar a mãe $(r=.16, p=.001)$. Por último, a autoconfiança correlacionase significativamente no sentido negativo com magnitude moderada com o medo de desapontar a mãe $(r$ $=-.21, p=.001)$.

Relativamente à associação entre as dimensões que constituem o ITEA na versão do pai, os resultados indicam a existência de correlações significativas. Assim sendo, destaca-se que a dimensão procura de suporte regista uma correlação significativa no sentido positivo e com magnitude forte com a ligação $(r=.77, p=.001)$ e com o medo de desapontar ao pai $(r=.55, p=.001)$ e uma correlação negativa e significativa de magnitude fraca com a intrusividade $(r=-.03, p=.001)$ e magnitude moderada com a autoconfiança $(r=-.41, p=.001)$. Por sua vez, a ligação correlaciona-se significativamente no sentido negativo com magnitude fraca com a intrusividade $(r=-.11, p=.001)$ e a autoconfiança $(r=-.27, p=.001)$ e no sentido positivo com magnitude moderada com o medo de desapontar o pai $(r=.50, p=.001)$. Relativamente à intrusividade, esta revela uma associação significativa no sentido positivo com magnitude fraca com a autoconfiança $(r=.19, p=.001)$ e medo de desapontar o pai $(r=.16, p=.001)$. Por último, a autoconfiança correlaciona-se significativamente no sentido negativo com magnitude moderada com o medo de desapontar o pai $(r=-.24, p=.001)$.

Tabela 3. Matriz de correlações Pearson do ITEA e suas dimensões

\begin{tabular}{|c|c|c|c|c|c|c|c|c|c|c|}
\hline Variáveis & 1 & 2 & 3 & 4 & 5 & 6 & 7 & 8 & 9 & 10 \\
\hline \multicolumn{11}{|l|}{ ITEA_MÃE } \\
\hline 1. Procura de suporte & 1 & - & & & & & & & & \\
\hline 2. Ligação & $.73^{* *}$ & 1 & - & & & & & & & \\
\hline 3. Intrusividade & -.03 & $-.15^{* *}$ & 1 & - & & & & & & \\
\hline 4. Autoconfiança & $-.36^{* *}$ & $-.18^{* *}$ & $.20^{* *}$ & 1 & - & & & & & \\
\hline 5. Medo de desapontar os pais & $.54^{* *}$ & $.46^{* *}$ & $.16^{* *}$ & $-.21^{* *}$ & 1 & & & & & \\
\hline \multicolumn{11}{|l|}{ ITEA_PAI } \\
\hline 6. Procura de suporte & $.48^{* *}$ & $.35^{* *}$ & $-.17^{* *}$ & $-.24^{* *}$ & $.16^{* *}$ & 1 & - & & & \\
\hline 7. Ligação & $.33^{* *}$ & $.45^{* *}$ & $-.24^{* *}$ & $-.11^{* *}$ & $.13^{* *}$ & $.77^{* *}$ & 1 & - & & \\
\hline 8. Intrusividade & $-.06^{* *}$ & $-.17^{* *}$ & $.75^{* *}$ & $.21^{* *}$ & $.04^{* *}$ & $-.03^{* *}$ & $-.11^{* *}$ & 1 & - & \\
\hline 9. Autoconfiança & $-.29^{* *}$ & $-.11^{* *}$ & $.25^{* *}$ & $.86^{* *}$ & $-.11^{* *}$ & $-.41^{* *}$ & $-.27^{* *}$ & $.19^{* *}$ & 1 & - \\
\hline 10. Medo de desapontar os pais & $.33^{* *}$ & $.23^{* *}$ & $.08^{* *}$ & $-.10^{* *}$ & $.64^{* *}$ & $.55^{* *}$ & $.50^{* *}$ & $.16^{* *}$ & $-.24^{* *}$ & 1 \\
\hline $\mathrm{M}$ & 4.09 & 4.34 & 2.75 & 3.14 & 3.95 & 3.53 & 3.95 & 2.48 & 3.22 & 3.81 \\
\hline DP & .80 & .63 & .89 & .84 & .69 & .92 & .83 & .81 & .85 & .82 \\
\hline
\end{tabular}

\section{Estrutura de invariância}

Depois de verificar um bom ajustamento do modelo original do ITEA à amostra Portuguesa em estudo, analisou-se a invariância de medida do ITEA entre papéis parentais e o género do participante através do IBM SPSS AMOS 25. Para testar a invariância de medida foram analisados os seguintes modelos aninhados: invariância configural, invariância métrica, invariância escalar, invariância residual. A invariância configural avalia se os participantes de diferentes grupos conceptualizam os constructos de forma idêntica. A invariância métrica analisa se diferentes grupos respondem aos itens de forma idêntica, isto é, se os pontos fortes das relações entre os itens de uma dimensão específica são iguais em diferentes grupos. A invariância escalar analisa se as pontuações observadas estão relacionadas com as pontuações latentes, isto é, se os participantes que apresentam o mesmo resultado na variável observada obterão o 
mesmo resultado na variável latente independentemente do fator grupo. Por último, a invariância residual permite testar se o nível de erro de medição presente para cada item é igual entre grupos (Milfont \& Fischer, 2010; Tagliabue \& Lanz, 2014). A confirmação de invariância de instrumento releva-se importante, na medida em que assegura que as comparações entre os grupos são significativas, e que as diferenças encontradas refletem efetivamente as discrepâncias existentes entre os grupos (Cheung \& Lau, 2012).

A invariância da medida entre os papéis dos pais foi testada aplicando a versão modificada do modelo de unicidade correlacionada (Tagliabue \& Lanz, 2014) devido à não independência das observações.

Em primeiro lugar, testou-se a invariância entre cada dimensão do pai e da mãe, separadamente (e.g., Ligação à mãe e Ligação ao pai), tendo-se encontrado invariância para todas as dimensões entre a versão da mãe e do pai. Tendo por base estes resultados, testou-se posteriormente um modelo com as cinco dimensões da versão da mãe e do pai ao mesmo tempo, correlacionando-as entre si. Os resultados sugerem invariância residual do ITEA entre papéis parentais: $\left(\chi^{2} / \mathrm{df}=2.435 ; \Delta \mathrm{CFI}=.003 ; \Delta \mathrm{RMSEA}=\right.$ .001) (Tabela 4). Por fim, testou-se a invariância de medida quanto ao género de participantes neste modelo final encontrando-se, igualmente, invariância residual $\left(\chi^{2} / \mathrm{df}=1.937 ; \Delta \mathrm{CFI}=.004 ; \Delta \mathrm{RMSEA}=\right.$ .001) (Tabela 5).

Tabela 4. Modelo de unicidade correlacionada - estatísticas de invariância de medição para os papéis parentais

\begin{tabular}{lccccc}
\hline \multirow{2}{*}{ MODELOS } & \multicolumn{3}{c}{ PAPÉIS PARENTAIS } \\
\cline { 2 - 5 } & $\chi \mathbf{2 / d f}$ & CFI & RMSEA & $\boldsymbol{\Delta C F I}$ & $\boldsymbol{\Delta}$ RMSEA \\
\hline Invariância Configural & 2.357 & .959 & .056 & - & - \\
Invariância Métrica & 2.376 & .957 & .056 & .002 & .000 \\
Invariância Escalar & 2.403 & .957 & .057 & .000 & -.001 \\
Invariância Residual & 2.435 & .954 & .058 & .003 & .001 \\
\hline
\end{tabular}

Nota. Os nomes dos modelos são os nomes dados pelo software Amos 25.

$\Delta=$ Mudança relativamente ao modelo anterior.

Tabela 5. Modelo de unicidade correlacionada - estatísticas de invariância de medição para o género dos participantes

\begin{tabular}{lccccc}
\hline \multirow{2}{*}{ MODELOS } & \multicolumn{5}{c}{ GÉNERO DOS PARTICIPANTES } \\
\cline { 2 - 5 } & $\chi \mathbf{2} / \mathbf{d f}$ & CFI & RMSEA & \multicolumn{1}{c}{$\boldsymbol{\Delta C F I}$} & \multicolumn{1}{c}{ RMSEA } \\
\hline Invariância Configural & 1.897 & .940 & .046 & - & - \\
Invariância Métrica & 1.903 & .939 & .046 & .001 & .000 \\
Invariância Escalar & 1.924 & .938 & .046 & .001 & .000 \\
Invariância Residual & 1.937 & .936 & .047 & .002 & -.001 \\
\hline
\end{tabular}

Nota. Os nomes dos modelos são os nomes dados pelo software Amos 25.

$\Delta=$ Mudança relativamente ao modelo anterior.

\section{Análises diferenciais}

Com o objetivo de analisar as diferenças da individuação à mãe e ao pai em função das da idade e do género dos participantes em estudo, efetuaram-se análises de variância uni e multivariada (ANOVA e MANOVA).

No que respeita à variável idade, importa salientar que foram estabelecidos dois grupos (1- dos 18 aos 21 anos; 2- dos 22 aos 30 anos) para se proceder às análises diferenciais. Os resultados sugerem a presença de diferenças estatisticamente significativas face ao processo de individuação à mãe $F(5,421)=$ 10.07, $\mathrm{p}=.001, \eta^{2}=.99$ e ao pai $\mathrm{F}(5,410)=4.04, \mathrm{p}=.001, \eta^{2}=.95$. As análises univariadas revelam que esta diferenciação ocorre nas escalas procura de suporte à mãe $F(1,425)=26.21, \mathrm{p}=.001, \eta^{2}=.99$; ligação à mãe $\mathrm{F}(1,425)=16.28, \mathrm{p}=.001, \eta^{2}=.98$; medo de desapontar a mãe $\mathrm{F}(1,425)=31.98, \mathrm{p}=.001$, $\eta^{2}=.99$; e medo de desapontar o pai $\mathrm{F}(1,414)=11.06, \mathrm{p}=.001, \eta^{2}=.91$, nas quais o grupo dos 18 aos 21 anos evidencia níveis mais elevados comparativamente com o grupo dos 22 aos 30 anos. As análises univariadas apontam, também, para a presença de diferenças estatisticamente significativas nas escalas autoconfiança à mãe $F(1,425)=17.97, \mathrm{p}=.001, \eta^{2}=.99$; e autoconfiança ao pai $\mathrm{F}(1,414)=6.86, \mathrm{p}=.009$, 
$\eta^{2}=.74$, sendo o grupo dos 22 aos 30 anos o que evidencia níveis mais elevados de autoconfiança em relação a ambos os pais, quando comparado com o grupo dos 18 aos 21 anos (Tabela 6).

Face à variável género, os resultados apontam para a existência de diferenças estatisticamente significativas no processo de separação em relação à mãe $F(5,421)=12.81, p=.001, \eta^{2}=.99$ e em relação ao pai $F(5,410)=8.56, p=.001, \eta^{2}=.99$. A partir das análises univariadas foi possível constatar que esta diferenciação ocorre nas escalas procura de suporte à mãe $F(1,425)=34.68, p=.99, \eta^{2}=.84$; na procura de suporte ao pai $F(1,414)=12.46, p=.001, \eta^{2}=.94$; na ligação à mãe $F(1,425)=12.76, p=.001$, $\eta^{2}=.95$; na ligação ao pai $F(1,414)=7.08, p=.008, \eta^{2}=.76$; no medo de desapontar a mãe $F(1,425)=$ $29.49, p=.001, \eta^{2}=.99$, e no medo de desapontar o pai $F(1,414)=25.60, p=.001, \eta^{2}=.99$, nas quais se constatou níveis superiores no género feminino comparativamente com o género masculino. As análises univariadas apontam, ainda, para a presença de diferenças significativas nas escalas intrusividade à mãe $F(1,425)=4.89, p=.027, \eta^{2}=.60$; na autoconfiança à mãe $F(1,425)=30.31, p=.001, \eta^{2}=.99$; e na autoconfiança ao pai $F(1,414)=19.82, p=.001, \eta^{2}=.99$, nas quais o género masculino evidenciou níveis superiores comparativamente com o feminino.

Tabela 6. Análise diferencial do processo de individuação em função da idade e do género

\begin{tabular}{|c|c|c|c|c|}
\hline & Idade grupos & $M \pm D P$ & IC $95 \%$ & $\begin{array}{c}\text { Direção das diferenças } \\
\text { significativas }\end{array}$ \\
\hline \multicolumn{5}{|l|}{ ITEA_MÃE } \\
\hline \multirow{2}{*}{ Procura de suporte } & $1-18-21$ & $4.20 \pm .71$ & {$[4.12,4.29]$} & \multirow{2}{*}{$1>2$} \\
\hline & $2-22-30$ & $3.77 \pm .95$ & {$[3.62,3.91]$} & \\
\hline \multirow{2}{*}{ Ligação } & $1-18-21$ & $4.41 \pm .54$ & {$[4.34,4.80]$} & \multirow{2}{*}{$1>2$} \\
\hline & $2-22-30$ & $4.14 \pm .80$ & {$[4.02,4.25]$} & \\
\hline \multirow{2}{*}{ Autoconfiança } & $1-18-21$ & $3.03 \pm .81$ & {$[2.94,3.12]$} & \multirow{2}{*}{$1<2$} \\
\hline & $2-22-30$ & $3.41 \pm .85$ & {$[3.26,3.56]$} & \\
\hline \multirow{2}{*}{ Medo de desapontar } & $1-18-21$ & $4.05 \pm .62$ & {$[3.98,3.57]$} & \multirow{2}{*}{$1>2$} \\
\hline & $2-22-30$ & $3.64 \pm .79$ & {$[3.52,3.76]$} & \\
\hline \multicolumn{5}{|l|}{ ITEA_PAI } \\
\hline \multirow{2}{*}{ Autoconfiança } & $1-18-21$ & $3.15 \pm .84$ & {$[3.06,3.25]$} & \multirow{2}{*}{$1<2$} \\
\hline & $2-22-30$ & $3.40 \pm .87$ & {$[3.24,3.56]$} & \\
\hline \multirow{3}{*}{ Medo de desapontar } & $1-18-21$ & $3.89 \pm .82$ & {$[3.80,3.98]$} & \multirow{2}{*}{$1>2$} \\
\hline & $2-22-30$ & $2.59 \pm .79$ & {$[3.44,3.74]$} & \\
\hline & Género & $M \pm D P$ & IC $95 \%$ & $\begin{array}{c}\text { Direção das diferenças } \\
\text { significativas }\end{array}$ \\
\hline \multicolumn{5}{|l|}{ ITEA_MÃE } \\
\hline \multirow{2}{*}{ Procura de suporte } & 1- Masculino & $3.60 \pm .81$ & {$[3.43,3.78]$} & \multirow{2}{*}{$1<2$} \\
\hline & 2-Feminino & $4.19 \pm .76$ & {$[4.11,4.27]$} & \\
\hline \multirow{2}{*}{ Ligação } & 1- Masculino & $4.10 \pm .67$ & {$[3.96,4.24]$} & \multirow{2}{*}{$1<2$} \\
\hline & 2-Feminino & $4.39 \pm .61$ & {$[4.32,4.45]$} & \\
\hline \multirow{2}{*}{ Intrusividade } & 1-Masculino & $2.96 \pm .88$ & {$[2.76,3.16]$} & \multirow{2}{*}{$1>2$} \\
\hline & 2- Feminino & $2.71 \pm .89$ & {$[2.61,2.80]$} & \\
\hline \multirow{2}{*}{ Autoconfiança } & 1- Masculino & $3.61 \pm .72$ & {$[3.42,3.79]$} & \multirow{2}{*}{$1>2$} \\
\hline & 2-Feminino & $3.04 \pm .82$ & {$[2.95,3.12]$} & \\
\hline \multirow{2}{*}{ Medo de desapontar } & 1-Masculino & $3.56 \pm .70$ & {$[3.40,3.71]$} & \multirow{2}{*}{$1<2$} \\
\hline & 2-Feminino & $4.02 \pm .66$ & {$[3.95,4.09]$} & \\
\hline \multicolumn{5}{|l|}{ ITEA_PAI } \\
\hline \multirow{2}{*}{ Procura de suporte } & 1-Masculino & $3.18 \pm .92$ & {$[2.97,3.40]$} & \multirow{2}{*}{$1<2$} \\
\hline & 2- Feminino & $3.60 \pm .90$ & {$[3.50,3.70]$} & \\
\hline \multirow{2}{*}{ Ligação } & 1-Masculino & $3.71 \pm .94$ & {$[3.52,3.91]$} & \multirow{2}{*}{$1<2$} \\
\hline & 2- Feminino & $4.00 \pm .80$ & {$[3.91,4.09]$} & \\
\hline Autoconfiança & 1- Masculino & $3.62 \pm .74$ & {$[3.43,3.81]$} & $1>2$ \\
\hline
\end{tabular}




\section{DISCUSSÃO}

0 presente estudo teve como principal objetivo adaptar para a população portuguesa o ITEA e analisar as suas características psicométricas. Foi, igualmente, objetivo deste estudo analisar a invariância do ITEA entre os papéis parentais e o género dos participantes.

Esta investigação fornece suporte relativamente ao ajustamento da estrutura fatorial do ITEA na amostra Portuguesa em estudo, assim como evidência quanto à sua consistência interna e invariância de medida.

Os resultados obtidos na AFC de 1. a ordem revelam que a estrutura original do instrumento de cinco fatores se replica na amostra em estudo, confirmando a sua adequação cultural e linguística à realidade portuguesa face ao significado atribuído pelos autores originais. Neste sentido, foram mantidos todos os itens e replicada a estrutura fatorial do ITEA, destacando-se este instrumento como uma medida fiável para se analisar o processo de individuação dos jovens adultos portugueses face às figuras parentais (Fiel, 2005; Kline, 2005). Desta forma, confirma-se que a versão portuguesa do ITEA revela coeficientes psicométricos equivalentes aos da versão original do instrumento (Komidar et al., 2013), não sendo possível realizar qualquer análise de comparação com níveis do seu ajustamento a outras culturas, dada a inexistência de evidência empírica.

Os resultados observados realçam a presença de valores satisfatórios de consistência interna em todas as dimensões, assim como na escala global, o que indica que os 36 itens que constituem o ITEA apresentem uma correlação aceitável. Desta forma, sugere-se que o conteúdo de todos os itens se aproxime do objetivo central do ITEA, o que confirma a sua capacidade para avaliar o processo de individuação à mãe e ao pai na amostra em estudo. A presença destes resultados sustenta a adequação do ITEA para fins de investigação científica (e.g., Nunnally \& Bernstein, 1994) indo ao encontro dos dados obtidos na versão original do instrumento (Komidar et al., 2013).

Relativamente às correlações entre as dimensões do ITEA na versão da mãe e do pai, estas revelaram-se significativas, ressaltando-se que o sentido e magnitude das associações se replicaram na versão de ambas as figuras parentais. Neste sentido, sugere-se que os cinco fatores de primeira ordem que compõem o ITEA se comportem entre si na amostra portuguesa, de forma similar à da versão original (Komidar et al., 2013).

Para além disso, este estudo estabelece evidências importantes sobre a invariância do ITEA. Embora a invariância de medição seja um pré-requisito para a realização de comparações entre grupos, raramente é testada (Lee, Tsai, Chiu, \& Ho, 2016). Assim, a falta de variação do ITEA entre os papéis parentais, bem como entre o género dos participantes, sugere uma evidência importante para a robustez dessa medida de avaliação do processo de individuação aos pais em jovens adultos. Comparações significativas de estatísticas, como por exemplo as médias, só podem ser feitas se um instrumento de medição avaliar o mesmo constructo entre diferentes grupos. Dessa forma, as evidências de invariância obtidas neste estudo garantem que: a) as comparações entre o pai e mãe e entre os géneros são significativas; b) o instrumento avalia o mesmo constructo em ambos os grupos; c) e que as diferenças entre os grupos refletem efetivamente as diferenças existentes entre eles (Cheung \& Lau, 2012). Este resultado assume relevância na medida em que muitos estudos que usam o ITEA compararam as respostas dos participantes em relação à individuação ao pai e à mãe, e entre os géneros. Os resultados observados asseguram que as estas comparações são possíveis e que as conclusões subsequentes são confiáveis. Estes resultados corroboram em parte a evidência empírica prévia, na medida em que no estudo original do ITEA foi encontrada invariância escalar entre o género dos participantes (Komidar et al., 2013). Mais recentemente foi testada a invariância de medida da versão breve do ITEA quanto aos papéis parentais, tendo sido apurada invariância escalar (Komidar, Zupančič, Levpušček, \& Bjornsen, 2016).

De destacar, ainda, que o ITEA se revelou capaz de identificar diferenças no processo de distanciamento psicológico aos pais por parte dos jovens adultos em função das variáveis sociodemográficas em estudo, nomeadamente idade e género. Constatou-se que os jovens adultos, dos 18 aos 21 anos, revelaram níveis superiores de procura de suporte e ligação à mãe e medo de desapontar ambos os pais, comparativamente com os jovens com idades compreendidas entre os 22 e os 30 anos. De notar que a literatura sugere que é nesta idade, entre os 18 e os 21 anos, que os jovens adultos estão mais dependentes da figura significativa, pelas tomadas de decisões relativamente às suas preferências e opções, desenvolvendo sentimentos de apreensão em não corresponder às expetativas das figuras parentais (e.g., Buhl, 2008). Segundo Zupančič e Kavčič (2014), os jovens adultos tendem a revelar uma ligação mais consistente com a figura materna o que, por sua vez, poderá potenciar um maior receio face à sua incapacidade de corresponder às expectativas desta figura.

Para além disto, verificou-se que os jovens adultos com idades entre os 22 e os 30 anos apresentaram níveis superiores de autoconfiança em relação à mãe e ao pai, comparativamente com os 
jovens entre os 18 e os 21 anos. Estes resultados vão ao encontro da perspetiva de Zupančič e Kavčič (2014) que considera que a independência dos adultos emergentes ocorre e consolida-se com o avançar da idade. Koepke e Denissen (2012) realçam, ainda, que a consolidação dos compromissos e escolhas se associam com o aumento da idade e maturidade atingida, o que pode consentir a diminuição dos cuidados parentais e aumento da autonomia e individualidade.

Por último, verificou-se que o género feminino revelou níveis superiores de procura de suporte, ligação e medo de desapontar face a ambas as figuras parentais, enquanto o género masculino revelou índices mais elevados de intrusividade face à mãe e autoconfiança relativamente a ambos os pais. Resultados similares são reportados pela evidência empírica que sublinha esta desigualdade na vivência e subjetividade do processo de individuação entre rapazes e raparigas (e.g., Zupančič \& Kavčič, 2014).

Note que os resultados encontrados nesta amostra vão ao encontro da literatura científica (e.g., Koepke \& Denissen, 2012) e corroboram a evidência empírica precedente (e.g., Komidar et al., 2013; Zupančič \& Kavčič, 2014), o que sugere a adequação cultural e linguística do ITEA para avaliar o processo de individuação em jovens adultos portugueses.

Como referências finais, cabe elencar as principais implicações práticas alcançadas com a concretização deste estudo, bem como, apontar as limitações que lhe estão subjacentes. Desta forma, realça-se que os resultados obtidos confirmam a abordagem teórica do processo de individuação da adultícia emergente na população portuguesa mediante a progressiva reestruturação das relações afetivas com os pais reformulando-as em interações entre adultos iguais. A relevância desta investigação acresce, ainda, pelo facto de ter contribuído para o desenvolvimento da investigação empírica no âmbito do processo de separação-individuação aos pais, mediante a apresentação de um modelo adequado e ajustado à cultura portuguesa para a avaliação deste processo psicológico em jovens adultos. Desta forma, sugere-se que a versão portuguesa do ITEA possa contribuir para o desenvolvimento de intervenções longitudinais, que possibilitem uma análise continuada do processo de separação-individuação aos pais ao longo da jovem adultícia e, assim melhor compreender o seu ajustamento psicoafetivo.

Apesar do seu contributo, algumas limitações estão subjacentes ao presente estudo. Cabe destacar que embora seja consensual na comunidade científica, que o modelo de equações estruturais estabelece um procedimento estatístico que permite assegurar a qualidade dos coeficientes psicométricos de um instrumento de medida de um constructo psicológico (e.g., Kline 2005), também é sabido que a generalização de um modelo considerado ajustado numa amostra para a restante população deverá ser criteriosa (e.g., Byrne \& Watkins, 2003). Para além disto, salienta-se que o estudo das características psicométricas do ITEA foi realizado numa amostra de estudantes universitários mediante dados transversais. Desta forma, sublinha-se a importância de se desenvolver novas investigações considerando uma amostra mais representativa de adultos emergentes portugueses que possibilitem testar a estabilidade da estrutura fatorial do ITEA, assim como, analisar a validade deste instrumento. Neste seguimento, destaca-se que o presente estudo apresenta apenas indicadores internos da validade do ITEA, pelo que se considera fundamental em estudos posteriores analisar a validade de constructo divergente e convergente e a validade preditiva do instrumento face a outras variáveis. Considera-se que a realização de tais análises permitiria a formulação de conclusões mais rigorosas acerca do processo de separação-individuação aos pais dos adultos emergentes, podendo contribuir para melhor compreender o seu ajustamento psicoafectivo. Apesar disto, salienta-se que o presente estudo apresenta indicadores importantes acerca das propriedades psicométricas do ITEA, no sentido de avaliar o processo de individuação-separação face às figuras parentais na jovem adultícia.

\section{REFERÊNCIAS}

Arnett, J. (2007). Emerging adulthood: what is it, and what is it good for? Child Development Perspectives, 1(1), 68-73. http://dx.doi.org/10.1111/j.1750-8606.2007.00016.x

Barrett, P. T., \& Kline, P. (1981) Radial parcel factor analysis. Personality and Individual Differences, 2, 311318. http://dx.doi.org/ 10.1016/0191-8869(81)90086-6

Blos, P. (1967). The second individuation process of adolescence. The Psychoanalytic Study of the Child, 22(1), 162-186. http://dx.doi.org/ 10.1080/00797308.1967.11822595

Buhl, H. M. (2008). Significance of individuation in adult child-parent relationships. Journal of Family Issues, 29(1), 262-281. http://dx.doi.org/ 10.1177/0192513X07304272

Buhl, H. M., \& Lanz, M. (2007). Emerging adulthood in Europe: common traits and variability across five European countries. Journal of Adolescent Research, 22, 439-443. http://dx.doi.org/ $10.1177 / 0743558407306345$

Byrne, B. M., \& Watkins, D. (2003). The issue of measurement invariance revisited. Journal of CrossCultural Psychology, 34(2), 155-175. http://dx.doi.org/ 10.1177/0022022102250225 
Cheung, G. W., \& Lau, R. S. (2012). A direct comparison approach for testing measurement invariance. Organizational Research Methods, 15(2), 167-198. http://dx.doi.org/ $10.1177 / 1094428111421987$

Coffman, D. L., \& MacCallum, R. C. (2005). Using parcels to convert path analysis models into latent variable models. Multivariate Behavioral Research, 40, 235-259. http://dx.doi.org/10.1207/s15327906mbr4002_4

Correia, F., \& Mota, C. P. (2016). Ambiente familiar e qualidade da vinculação amorosa - papel mediador da individuação em jovens adultos. Análise Psicológica, 1(34), 15-29. http://dx.doi.org/10.14417/ap.1018.

Cronbach, L. J. (2004). My current thoughts on coefficient alpha and successor procedures. Educational and Psychological Measurement, 64, 391-418. http://dx.doi.org/10.1177/0013164404266386

Field, A. (2005). Discovering statistics using SPSS. London: Sage Publications.

Hambleton, R. K. (2005). Issues, designs and technical guidelines for adapting tests into multiple languages and cultures. In R. K. Hambleton, P. F. Merenda, \& C. D. Spielberger (Eds.), Adapting educational and psychological tests for cross-cultural assessment (pp. 3-38). Mahwah, NJ: Erlbaum.

Josselson, R. (1980). Ego development in adolescence. In J. Adelson (Ed.), Handbook of adolescent psychology (pp. 188-210). New York: Wiley.

Klein, R. B. (2005). Principles and practice of structural equation modeling. New York \& London: The Guilford Press.

Koepke, S. \& Denissen, J. (2012). Dynamics of identity development and separation-individuation in parent-child relationships during adolescence and emerging adulthood - a conceptual integration. Developmental Review, 32, 67-88. http://dx.doi.org/10.1016/j.dr.2012.01.001

Komidar, L., Zupančič, M., Puklek Levpušček, M., \& Bjornsen, C. A. (2016). Development of the short version of the Individuation Test for Emerging Adults (ITEA-S) and its measurement invariance across Slovene and U.S. emerging adults. Journal of Personality Assessment, 98(6), 626-639. http://dx.doi.org/10.1080/00223891.2016.1171231.

Komidar, L., Zupančič, M., Sočan, G., \& Puklek Levpušček, M. (2013). Development and construct validation of the individuation test for emerging adults (ITEA). Journal of Personality Assessment, 96, 503-514. http://dx.doi.org/10.1080/00223891.2013.850703.

Kruse, J., \& Walper, S. (2008). Types of individuation in relation to parents: predictors and outcomes. International Journal of Behavioral Development, 32, 390-400. http://dx.doi.org/10.1177/0165025408093657

Lamborn, S. D., \& Groh, K. (2009). A four-part model of autonomy during emerging adulthood: associations with adjustment. International Journal of Behavioral Development, 33, 395-401. http://dx.doi.org/10.1177/0165025409338440

Lee, K. L., Tsai, S. L., Chiu, Y. T., \& Ho, M. J. (2016). Can student self-ratings be compared with peer ratings? A study of measurement invariance of multisource feedback. Advances in Health Sciences Education, 21(2), 401-413. http://dx.doi.org/10.1007/s10459-015-9638-5

Levine, S. Z., Petrides, K. V., Davis, S. Jackson, C. J., \& Howell, P. (2005). The use of structural equation modelling in stuttering research: Concepts and directions. Stammering Research, 1, 344-363.

MacCallum, R. C., Widaman, K. F., Preacher, K. J., \& Hong, S. (2001). Sample size in factor analyses: the role of model error. Multivariate Behavioral Research, 36, 611-637. http://dx.doi.org/10.1207/S15327906MBR3604_06

Mahler, M., Pine, F., \& Bergman, A. (1975). The psychological birth of human infant. London \& United Kingdom: Hutchinson.

Milfont, T. L., \& Fischer, R. (2010). Testing measurement invariance across groups. Applications in crosscultural research. International Journal of Psychological Research, 3(1), 111-121. http://dx.doi.org/10.21500/20112084.857

Mota, C. P., \& Matos, P. M. (2013). Conflitos interparentais e individuação em jovens adultos portugueses: Papel dos conflitos de lealdade. Psicologia: Teoria e Pesquisa, 29(3), 83-92. http://dx.doi.org/10.1590/S0102-37722013000300004

Nunnally, J. C., \& Bernstein, I. H. (1994). Psychometric theory (3 ${ }^{\text {rd }}$ ed.). New York: McGraw-Hill.

Puklek Levpušček, M. (2006). Adolescent individuation in relation to parents and friends: age and gender differences. European Journal of Developmental Psychology, 3, 238-264. http://dx.doi.org/10.1080/17405620500463864

Tagliabue, S., \& Lanz, M. (2014). Exploring social and personal relationships: The issue of measurement invariance of non-independent observations. European Journal of Social Psychology, 44(7), 683690. https://doi.org/10.1002/ejsp.2047 
Tanner, J. L. (2006). Recentering during emerging adulthood: a critical turning point in life span human development. In J. Arnett \& J. L. Tanner (Eds.), Emerging adults in America (pp. 21-55). Washington: American Psychological Association.

Walper, S., Schwarz, B., \& Jurasic, S. (1996). Entwicklung und erprobung des münchner individuations tests. Berichte aus der arbeitsgruppe "aamilienentwicklung nach der trennung" 8/1996. LudwigMaximilians: Universität Munchen Press.

Youniss, J., \& Smollar, J. (1985). Adolescent relations with mothers, fathers, and friends. Chicago: University of Chicago Press.

Zupančič, M., \& Kavčič, T. (2014). Student personality traits predicting individuation in relation to mothers and fathers. Journal of Adolescence, 37, 715-726. http://dx.doi.org/10.1016/j.adolescence.2013.12.005.

Historial do artigo

Recebido $\quad 06 / 18$

Aceite $\quad 05 / 19$

Publicado $\quad 08 / 19$ 
Individuação em adultos emergentes 